algebraic geometry. The transparency of style makes the book excellent for use as a text or for the practicing mathematician who wishes to absorb the essentials of the theory quickly and painlessly. The expert will be especially grateful for the unified presentation of results which, except for the more elementary parts already available in several texts, have thus far appeared only in Ergebnisse-style monographs or in the many widely scattered original papers of the last 25 years.

The chapter headings are: I. The primary decomposition. II. Residue rings and rings of quotients. III. Some fundamental properties of Noetherian rings. (This includes Krull's intersection theorem, symbolic powers of prime ideals, composition series for primary ideals, and dimension theory.) IV. The algebraic theory of local rings. (Including accounts of regular local rings and the quasigleichheit theory.) V. The analytic theory of local rings. (The existence of a local ring's completion and some transition properties.) The book ends with brief notes indicating the history and applications of the theory. The applications indicated are of course to algebraic geometry, but the author quite naturally refrains from identifying the two subjects.

Polynomial rings appear only incidentally or as examples and no mention is made of their unique factorization. Very little is said about rings of dimension one and even the theory of Dedekind rings, whose full development would have required about one extra page at the appropriate place, is omitted. Field theory, valuation theory, the structure theory of complete local rings, the important relations existing between the ideals of a ring and an integrally dependent over-ring, the Hilbert function, and unmixedness theorems are either missing entirely (presumably for reasons of space, connectedness and essentiality) or are mentioned in passing, in the notes at the end. There is a brief bibliography, most of whose items are made mainly of historical interest by the present tract. A brief list of the best (as opposed to the earliest) references would have been of more help to the unguided reader wishing to pursue the subject further.

MAXWELl RosenLICHT

Complexes linéaires. Faisceaux de complexes linéaires. Suites et cycles de complexes linéaires conjugués. By A. Charrueau. Paris, GauthierVillars, 1952. $84 \mathrm{pp}$.

This memoir, as far as new contributions to the theory of linear complexes are concerned, is based primarily on a series of notes of the author appearing in the Comptes Rendus (Paris) during 1948 
and 1949. These notes introduced the concept of a sequence $C_{i}$ of linear complexes in which $C_{j+2}$ is the reciprocal polar of $C_{j}$ with respect to $C_{j+1}$ for all $j$; and also the concept of a cycle of linear complexes which is defined as a closed sequence, that is, $C_{n+1}=C_{1}, C_{n+2}$ $=C_{2}$, where $n$ is the number of complexes in the cycle.

The first part of the work is devoted to a review of the classical theory of linear complexes. The second part is concerned with the study of pencils of linear complexes. This again is mostly a résumé of known facts. There is a discussion of the locus of the poles of planes passing through a fixed straight line with respect to all the complexes of a pencil; also a discussion of the straight lines conjugate to a fixed straight line with respect to all the complexes of a pencil. A good choice of coordinate system, used extensively by the author, results in simplifying the equation of any pencil of linear complexes and the equations of geometric configurations associated with the pencil.

The third part of the memoir is devoted to the study of sequences and cycles of linear complexes. In every pencil of linear complexes, $\Phi=\phi_{1}+\lambda \phi_{2}=0$, where the base complexes have real coefficients, and at least one of these is not special, there exists a sequence (or cycle) of linear complexes. Let $\lambda_{1}$ and $\lambda_{2}$ be the values of $\lambda$ corresponding to the special complexes of $\Phi$, and let $\lambda_{1} \neq \lambda_{2}$. If $\mu_{j}$ is the value of $\lambda$ corresponding to $C_{j}$, and if $\alpha_{j}=\left(\mu_{j}-\lambda_{1}\right) /\left(\mu_{j}-\lambda_{2}\right)$, then $\alpha_{j} \alpha_{j+2}=\alpha_{j+1}^{2}$ so that the $\alpha_{j}$ form a geometric progression with the common ratio $\alpha_{2} / \alpha_{1}$. A necessary and sufficient condition that a sequence be a cycle of length $p$ is that this common ratio be a primitive $p$ th root of unity. In case $\lambda_{1}=\lambda_{2}$, no cycle in a pencil is possible.

The most general collineation or dualistic transformation transforms a linear complex into a linear complex and a pencil of linear complexes into a pencil of linear complexes. Consider products of transformations by reciprocal polars relative to members of a pencil of linear complexes, $\Phi=\phi_{1}+\lambda \phi_{2}=0$, where $\phi_{1}$ and $\phi_{2}$ are nonspecial and their equations have real coefficients. If $\tau_{\mu_{1}}, \tau_{\mu_{2}}, \cdots$, $\tau_{\mu_{n}}, \cdots$ are the transformations by reciprocal polars relative to the nonspecial complexes $\mu_{1}, \mu_{2}, \cdots, \mu_{n}, \cdots$ of $\Phi$ corresponding to the values $\mu_{1}, \mu_{2}, \cdots, \mu_{n}, \cdots$ of $\lambda$, and if $T_{n}=\tau_{\mu_{1}} \tau_{\mu_{2}} \cdots \tau_{\mu_{n}}$ is the product of the transformations $\tau_{\mu_{1}}, \tau_{\mu_{2}}, \cdots, \tau_{\mu_{n}}$, then $T_{n}$ is a collineation if $n$ is even or a dualistic transformation if $n$ is odd. If $\lambda_{1} \neq \lambda_{2}$, the transformation $T_{n}$ for $n$ odd and $>1$ is equal to the transformation $\tau_{k_{n}}$, relative to the nonspecial complex $k_{n}$ where $k_{n}$ is defined by $\left(k_{n}-\lambda_{1}\right) /\left(k_{n}-\lambda_{2}\right)=\alpha_{1} \alpha_{3} \alpha_{5} \cdots \alpha_{n} / \alpha_{2} \alpha_{4} \cdots \alpha_{n-1}$. The collineation $T_{n}$ depends only on $a_{n}=\alpha_{1} \alpha_{3} \cdots \alpha_{n-1} / \alpha_{2} \alpha_{4} \cdots \alpha_{n}$ for $n$ even. The author is able to give a geometrical interpretation for $a_{n}$. 
The case $\lambda_{1}=\lambda_{2}$ is discussed in a similar manner.

There is a brief discussion of the case where the parameters of all the members of a pencil are equal, the case where the axes of the base complexes of a pencil are parallel, and the case where the axes of all members of a pencil are identical.

The memoir is well printed with only a few minor misprints. It seems to this reviewer that the first two parts of the memoir are unnecessarily repetitive. The third part on cycles and sequences is interesting and is more compactly written.

Alice T. Schafer

\section{New Journal}

Journal d'Analyse Mathématique. Vols 1, 2. Jerusalem, 1951, 1952-53. Vol. $1,8+386+26$ pp.; vol. $2,8+381+23$ pp. $\$ 20$ per volume; $40 \%$ discount to individual members of mathematical societies.

Although there have been and still are a number of mathematical journals specializing in restricted fields, this is, I believe, the first to take analysis in general as its avowed specialty. As such, it is welcome to analysts, and should be welcome to other specialists at least for its effect in decreasing the pressure on other journals. The average length of the papers is appreciable: the two volumes contain 15 and 17 papers, respectively. Indeed, in a day when most authors are under pressure to emulate Landau in compression, although infrequently in clarity, of mathematical exposition, this journal is one of the last refuges of the long paper and the typographically complicated formula. One must be grateful to the editor, B. A. Amirà, for his courage, and to the organizations and individuals which have subsidized the publication so far. The journal is evidently going to be one of the indispensable components of any serious general mathematical library.

This is intended to be an international journal, and it has an international editorial board. In the first two volumes, an overwhelming majority of the papers originated in the U.S.A. or France. Both wellestablished names and relative newcomers appear in the list of authors. The languages of the journal are English and French; the extra pages at the ends of the volumes contain summaries in Hebrew. The typography of the formulas is excellent, but the pages have an unconventional look which can be traced to the wide spacing between the lines of the text and the use of spaced-out roman instead of italic type for statements of theorems and the like.

R. P. BOAS, JR. 